\title{
Observation of Rotation of Spin-Polarization with the Verwey Transition at a Surface of a $\langle 110\rangle$-Oriented Magnetite Whisker by Field-Emitted Electron Polarimetry*
}

\author{
S. Nagai ${ }^{\dagger}$ and K. Hata \\ Graduate School of Engineering, Mie University, 1577 Kurima-Machiya, Tsu 514-8507, Japan \\ M. Okada and H. Mimura \\ Research Institute of Electronics, Shizuoka University, 3-5-1 Johoku, Hamamatsu 432-8011, Japan
}

(Received 25 July 2011; Accepted 8 August 2011; Published 3 September 2011)

\begin{abstract}
We observed the Verwey transition, i. e., metal-insulator transition, in a spin-polarization of field-emitted electrons from a topmost surface of a $\langle 110\rangle$-oriented magnetite whisker by means of field-emitted electron polarimetry. With a rise in temperature of the whisker to about $120 \mathrm{~K}$, the polarization of field-emitted electrons increased from 4 to $11 \%$ with a rotation of its direction. The rotation angle corresponds to a change of magnetic easy axis from $\langle 001\rangle$ to $\langle 111\rangle$ direction caused by the Verwey transition. This result reveals that the Verwey transition occurs even at a surface as well as in a bulk.
\end{abstract}

[DOI: 10.1380/ejssnt.2011.322]

Keywords: Field emission microscopy; Field emission; Magnetic phenomena; iron oxide; Electron spin polarization

\section{INTRODUCTION}

Magnetite is a magnetic material which mankind acquired for the first time and has been investigated by many researchers from a long time ago. Nowadays, magnetite has theoretically been predicted to have perfect spin polarization at Fermi level $[1,2]$, i.e., half metallic property, and its spintronic applications are expected. The Verwey transition has been known as a phenomenon, which conductivity and magnetization of magnetite increase abruptly above transition temperature, i.e., the Verwey point $\left(T_{\mathrm{v}} \sim 120 \mathrm{~K}\right)[3,4]$. Simultaneously, the magnetic easy axis is rotated from $\langle 001\rangle$ to $\langle 111\rangle$ direction as temperature is raised. Magnetite $\mathrm{Fe}_{3} \mathrm{O}_{4}$ is $\mathrm{Fe}^{3+}\left(\mathrm{Fe}^{3+} \mathrm{Fe}^{2+}\right) \mathrm{O}_{4}^{2-}$, and has inversed spinel structure in which $\mathrm{Fe}^{3+}$ ion is located at the center of a tetrahedron with vertex of $\mathrm{O}^{2-}$ ions, i.e., A-site, while mixed valence $\mathrm{Fe}^{2.5+}$ ion is located at the center of an octahedron, i.e., B-site [5]. Verwey interpreted that transition is caused by a charge disordering between $\mathrm{Fe}^{2+}$ and $\mathrm{Fe}^{3+}$ ions located at the $\mathrm{B}$ site. In short, he set up a model that an extra electron included in $\mathrm{Fe}^{2+}$ ion behaves as free electron within the B site, and the electron is localized below Verwey point, and itinerates above it. Verwey advanced that $\mathrm{Fe}^{2+}$ and $\mathrm{Fe}^{3+}$ ions order alternately, but the model was disclaimed by experimental results of nuclear magnetic resonance and neutron diffraction method [6]. Recently, unifying model for an array of charge and an electronic structure has not yet been determined though it has become possible recently to directly show their detail by using spin-resolved scanning tunneling microscopy [7] and photoemission spectroscopy [8-10]. By these methods, it has been recognized that the transition come from the electronic state at Fermi level.

In these reports mentioned above, only bulky magnetite was investigated. However, it has been clarified whether

\footnotetext{
*This paper was presented at 8th International Symposium on Atomic Level Characterizations for New Materials and Devices, Olympic Parktel, Seoul, Republic of Korea, 22-27 May, 2011.

$\dagger$ Corresponding author: nagai@elec.mie-u.ac.jp
}

the transition occurs even at surface. For the purpose of clarification, we have suggested field-emitted electrons polarimetry, which is predicted that a spin polarization of field-emitted electrons from magnetic materials is proportional to the spin polarization around Fermi level at the topmost surface of the materials [11]. Therefore, the measurements of spin polarization of field-emitted electrons may help us to get knowledge about the spin polarization at the topmost surface. On our previous study [12, 13], measurements of spin polarization of field-emitted electrons from a $\langle 110\rangle$-oriented magnetite whisker was restricted to in-plane components perpendicular to emitter axis due to a lack of any spin rotator. Therefore, we developed a $90^{\circ}$ sector type spin rotator to rotate the spin vector before electrons enter to the Mott analyzer. Present paper describes all components of the spin polarization of field-emitted electrons from a magnetite whisker in the temperature around the Verwey point.

\section{EXPERIMENTAL SETUP}

Figure 1 shows a schematic diagram of a column of field emission microscope equipped with a spin rotator and a Mott spin polarization analyzer, whose pressure of $3 \times 10^{-8} \mathrm{~Pa}$. An emitter was attached to the cold finger of a He cooling machine. The cold finger and a ceramic heater permit a variation of the emitter temperature in a range from 40 to $300 \mathrm{~K}$. The emitter temperature was measured by chromel-gold/iron thermocouple. FEM image of the emitter was projected onto a fluorescent screen with a centered probe hole.

Electron optics for the measurements of spin polarization consists of four parts, two einzel lenses, the spin rotator, an einzel lens and the Mott spin polarization analyzer [14]. Firstly, two eintzel lenses, the second einzel lens contains two octapoles for deflection and astigmatism correction, focus and transfer the electron beam onto an objective position of the spin rotator. Secondly, a $E \times B$ deflector, as a spin rotator, is inserted between einzel lenses. The spin rotator geometry is that a $90^{\circ}$ spherical deflector with deflection radius of $50 \mathrm{~mm}$ is inserted into a gap of the magnetic polepieces at a ground potential. With an 


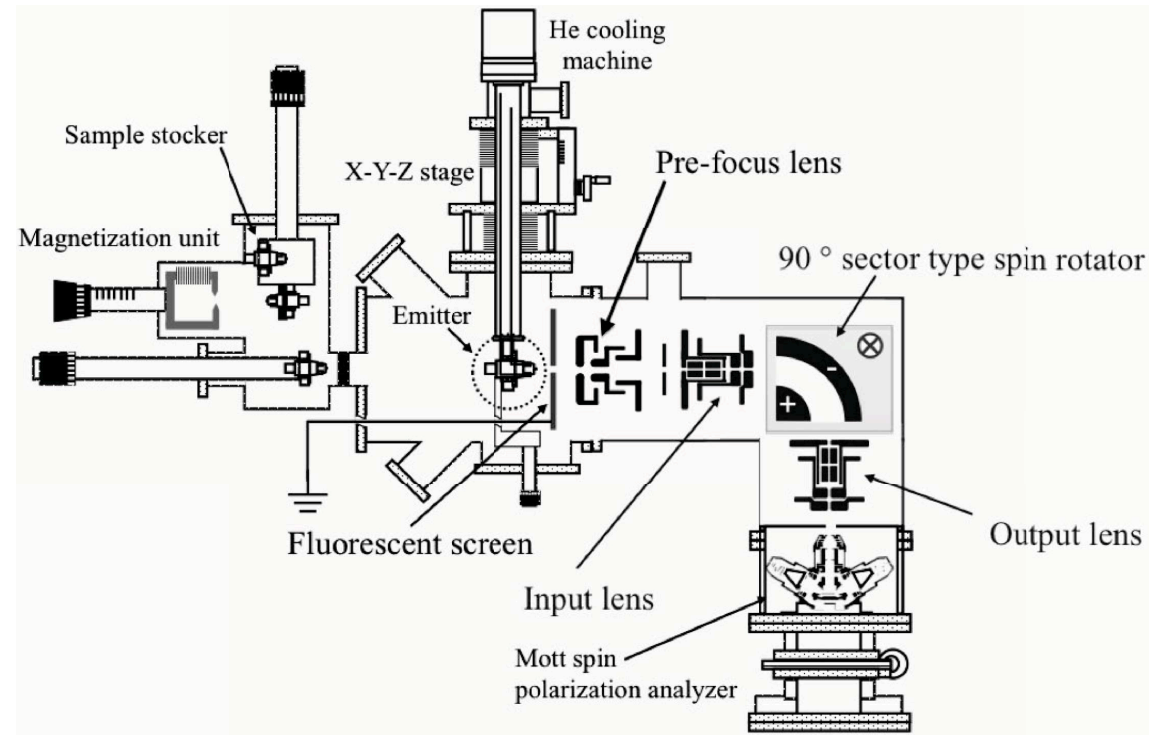

FIG. 1: Schematic diagram of a field emission microscope equipped with $90^{\circ}$ sector type spin rotator and retarding type spin polarization analyzer.

electrostatic deflection, the spin polarization vector is not affected. Thus, the $Z$ and $Y$ components of the spin polarization can be measured by the Mott spin polarization analyzer. On the other hand, with a magnetic deflection, the spin polarization vector of the emerging electrons is rotated at angle of $90^{\circ}$. Therefore, the $X$ and $Y$ components can be measured. By switching electrostatic or magnetic deflection, it is possible to detect all components of the spin polarization vector. Thirdly, an output lens makes the electron beam focused onto an entrance aperture of the Mott spin polarization analyzer. Finally, the entered electrons into the Mott analyzer are accelerated up to $25 \mathrm{keV}$ toward a scattering target. Electrons scattered by a gold target with thickness of $100 \mathrm{~nm}$ are detected by 4-channel electron multipliers (CEMs) arranged in 4-fold symmetry. Retarding grids, placed in front of the each CEM, block inelastic electrons that lost the kinetic energy higher than $600 \mathrm{eV}$. Asymmetry Am in the number of electrons detected by a pair of CEM's is defined by

$$
A_{\mathrm{m}}=\frac{N_{\mathrm{R}}-N_{\mathrm{L}}}{N_{\mathrm{R}}-N_{\mathrm{L}}}
$$

where, $N_{\mathrm{R}}$ and $N_{\mathrm{L}}$ are the numbers of electrons detected by the right and the left CEMfs respectively. Actually measured asymmetry $A_{\mathrm{m}}$ is summation of spin dependent and instrumental asymmetries, $A_{\mathrm{s}}$ and $A_{\mathrm{i}}$, respectively. Instrumental asymmetry $A_{\mathrm{i}}$ is caused by efficiency of the detector, irregularity of the target surface, incident angle of the beam axis etc. We defined $A_{\mathrm{i}}$ as asymmetry measured with respect to non-polarized electron from tungsten field emitter. Spin polarization $P$ and spin dependent asymmetry $A_{\mathrm{s}}$ are related by using an effective Sherman function $S_{\text {eff }}$ as

$$
P=\frac{A_{\mathrm{s}}}{S_{\mathrm{eff}}}=\frac{A_{\mathrm{m}}-A_{\mathrm{i}}}{S_{\mathrm{eff}}} .
$$

In this experiment, we used $S_{\text {eff }}$ of 0.15 reported by Iori [15] under the same experimental conditions as we

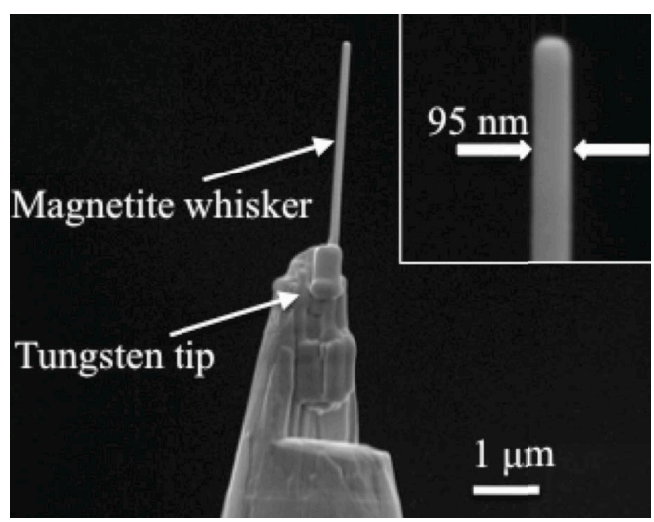

FIG. 2: Scanning electron micrograph of single magnetite emitter.

employed. $P_{X}, P_{Y}$ and $P_{Z}$ are defined to be the polarization of $X, Y$ and $Z$ components, respectively. Magnitude of spin polarization $P_{\text {total }}$, polar angle $\theta$ with respect to $Z$-direction and azimuthal angle on $\mathrm{X}$-Y plane $\phi$ with respect to $X$-direction are estimated by

$$
\begin{aligned}
P_{\text {total }} & =\left(P_{X}+P_{Y}+P_{Z}\right)^{1 / 2}, \\
\phi & =\arccos \left(P_{Z} / P_{\text {total }}\right),
\end{aligned}
$$

and

$$
\theta=\arctan \left(P_{Y} / P_{Z}\right)
$$

respectively.

\section{SAMPLE PREPARATION}

The magnetite whiskers were synthesized by thermal oxidation of a stainless steel substrate in air [16]. By means of electron diffraction with a transmission microscope (TEM), it was observed that the magnetite whiskers 


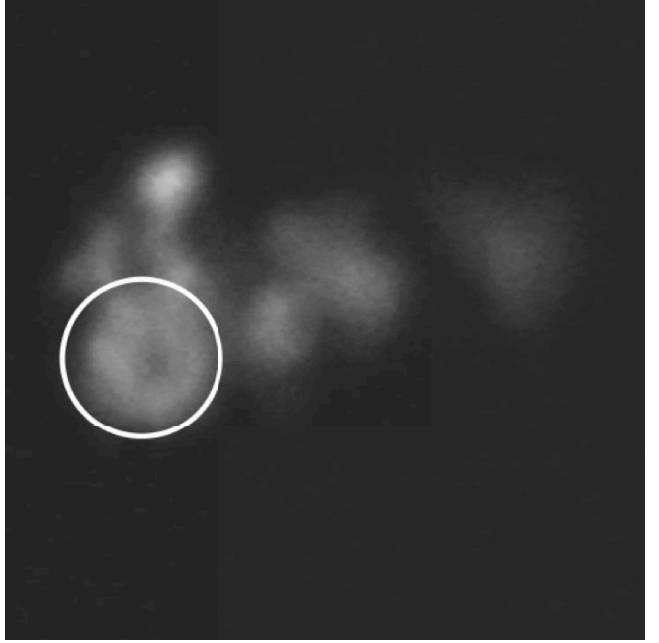

FIG. 3: FEM image of the magnetite whisker after the surface cleaning, which was obtaned at the voltage of $-1.3 \mathrm{kV}$ and total emission current of $23 \mathrm{nA}$.

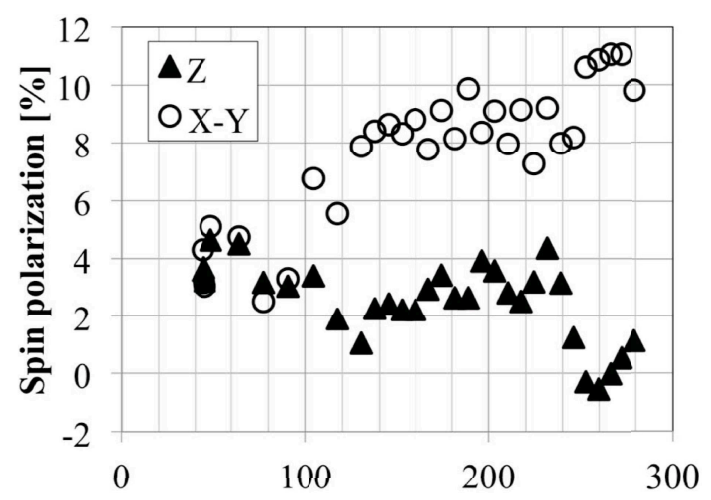

Tip temperature [K]

FIG. 4: Emitter temperature dependence of spin polarization of field emitted electrons from the magnetite whisker. Closed triangles indicate $Z$-component. Open circles indicate $X-Y$ plane components perpendicular to emitter axis.

were extending along $\langle 110\rangle$. In addition, according to energy dispersion X-ray spectroscopy (EDS), concentration of impurities, mainly manganese, was less than $1 \%$ [17]. One of whiskers was picked up and fixed on an electrochemically etched tungsten wire in a focused ion beam apparatus, as shown in Fig. 2. Before measurements, the emitter surface was cleaned by an annealing at $1200 \mathrm{~K}$ under a pressure of $3 \times 10^{-8} \mathrm{~Pa}$. It was confirmed that the annealing did not affect its stoichiometry and crystal structure by means of EDS and electron diffraction patterns in TEM. Following the annealing, field evaporations at voltage of $8.5 \mathrm{kV}$ were repeated until FEM images were identified before and after cleaning. The FEM image with fine structure after the cleaning procedure is shown in Fig. 3. The measurement of spin polarization was carried out with encircled emission site in Fig. 3, under the condition of total emission current of $23 \mathrm{nA}$ and applied voltage of $1.3 \mathrm{kV}$.

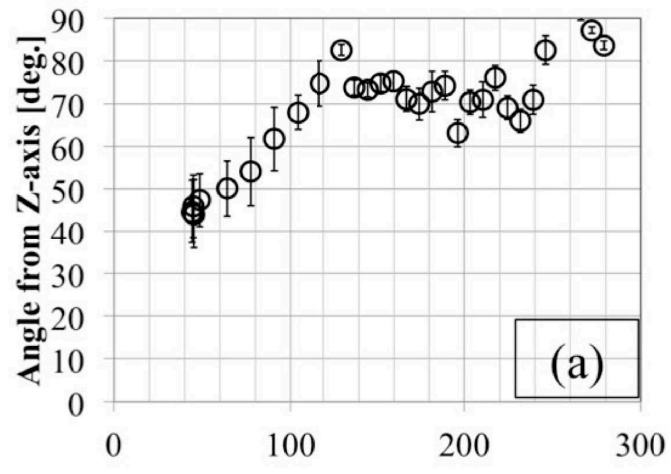

Tip temperature $[\mathbf{K}]$

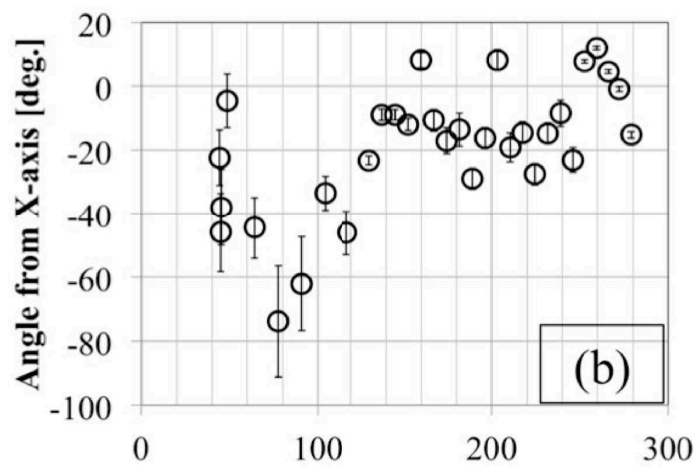

Tip temperature $[\mathrm{K}]$

FIG. 5: Temperature dependences of direction of spin polarization. (a) Polar angle with respect to $Z$-axis. (b) Azimuthal angle with respect to $X$-axis.

\section{RESULTS AND DISCUSSIONS}

Figure 4 shows spin polarizations of $X-Y$ and $Z$ components against emitter tip temperature ranging from 40 to $300 \mathrm{~K}$. At low temperature of $40 \mathrm{~K}$, a value of $X-Y$ component of polarization was about $4 \%$. The polarization increased gradually with the temperature from $100 \mathrm{~K}$, eventually reached about $11 \%$ at room temperature. This trend is similar to temperature dependence of magnetization observed with bulky magnetite [3], expecting that the observed transition was second-order transition. The second-order transition may be caused by impurity of Mn less than $1 \%$, which has been also observed with bulky mangetite [18]. On the other hand, the spin polarization of $Z$ component was below $5 \%$ with fluctuations, which was independent on the emitter temperature. Note that the spin polarization of $X-Y$ components was higher than that of $Z$ direction regardless its temperature. If shape anisotropy due to cylindrical shape determine magnetic property, $Z$-directional component may be the dominant component of spin polarization. But, in present study, the in-plane spin polarization dominated in total spin polarization. Thus it is considered that magnetic property for single-crystalline magnetite whisker was resulted from crystalline anisotropy rather than shape anisotropy.

Figures 5(a) and (b) shows temperature dependence of the direction of spin polarization for a polar angle $\theta$ with respect to $z$-axis and an azimuthal angle $\phi$ with respect to $X$-axis, respectively. With the increase of tip temper- 
ature, the direction rotated at the polar angle from $-43^{\circ}$ to $-83^{\circ}$, as shown in Fig. 5(a). For the azimuthal angle at low temperature, although there are stastical deviations due to low spin polarization, the rotation of $45 \pm 15^{\circ}$ was observed, as shown in Fig. 5(b). Consider the rotation of the spin polarization taking into account that the whisker is extending along $\langle 110\rangle$ direction and magnetic easy axis rotates from $\langle 100\rangle$ to $\langle 111\rangle$ direction with a rise in temperature. The rotational angle of the magnetitc easy axis between $\langle 100\rangle$ and $\langle 111\rangle$ directions with raising temperature of magnetite are $45^{\circ}$ in (001) plane and $35.2^{\circ}$ in (110), which are corresponding to $\theta$ and $\phi$, respectively. The dependence of value as well as its direction of the spin polarization on temperature were corresponding to a change of magnetization and magnetic easy axis from $\langle 100\rangle$ to $\langle 111\rangle$ direction which was caused by the Verwey transition. Thus, it is revealed that the increase of the spin polarization is attributed from the Verwey transition of magnetite and the transition occurs even at surface as well as in a bulk.

\section{CONCLUSIONS}

In this study, we measured temperature dependences of spin polarization of field-emitted electrons from $\langle 110\rangle$ oriented magnetite whisker. Raising temperature of the whisker, the polarization increased from 4 to $11 \%$ with a rotation of its direction. The rotation angle corresponds to a change of magnetic easy axis from $\langle 001\rangle$ to $\langle 111\rangle$ direction caused by the Verwey transition. This result revealed that the Verwey transition occurs even at a surface as well as in a bulk.

\section{Acknowledgments}

This work has been supported by Grant-in aid for Scientific Research (B), No. 18360023 of the Japanese Ministry of Education, Culture, Sports, Science and Technology.
[1] Z. Zhang and S. Satpathy, Phys. Rev. B 44, 13319 (1991).

[2] P. Piekarz, K. Parlinski, and A. M. Oles, Phys. Rev. B 76, 165124 (2007).

[3] E. J. W. Verwey, Nature 144, 327 (1939).

[4] H. J. Williams and R. M. Bozorth, Rev. Mod. Phys. 25, 79 (1953).

[5] E. J. Verwey, P. W. Haaymann, and F. C. Romeijn, J. Chem. Phys. 15, 181 (1947).

[6] W. C. Hamilton, Phys. Rev. 110, 1050 (1958).

[7] Y. S. Dedkov, U.Rudiger, and G. Guntherodt, Phys. Rev. B 65, 064417 (2002).

[8] Y. Q. Cai, K. Nakatsuji, S. Ohno, T. Iimori, M. Yamada, and F. Komori, Surf. Rev. Lett. 9, 907 (2002).

[9] J. G. Tobin, S. A. Morton, S. W. Yu, G. D. Waddill, I. K. Schuller, and S. A. Chambers, J. Phys.: Condens. Matter 19, 315218 (2007).

[10] M. Fonin, R. Pentcheva, Y. S. Dedkov, M. Sperlich, D. V. Vyalikh, M. Scheffler, U. Rudiger, and G. Guntherodt, Phys. Rev. B 72, 104436 (2005).
[11] T. E. Feuchtwang, P. H. Cutler, and J. Schmit, Surf. Sci. 75, 401 (1978).

[12] S. Nagai, K. Hata, M. Okada, and H. Mimura, J. Vac. Sci. Technol. B 28, C2A24 (2010).

[13] S. Nagai, K. Hata, M. Okada, and H. Mimura, Appl. Surf. Sci. 25, 1058 (2009).

[14] G. C. Burnett, T. J. Monroe, and F. B. Dunning, Rev. Sci. Instrum. 65, 1893 (1994).

[15] K. Iori, K. Miyamoto, H. Narita, K. Sakamoto, A. Kimura, S. Qiao, K. Shimada, H. Namatame, and M. Taniguchi, Rev. Sci. Instrum. 77, 13101 (2006).

[16] M. Okada, S. Nagai, Y. Neo, K. Hata, and H. Mimura, Jpn. J. Appl. Phys. 48, 06FE07 (2009).

[17] M. Okada, S. Nagai, Y. Neo, K. Hata, and H. Mimura, Phys. Status Solidi C 7, 2574 (2010).

[18] V. A. M. Brabers, F. Walz, and Kronmuller, Phys. Rev. B 58, 14163 (1998). 